\title{
Spectral analyses of eighteen hot H-deficient (pre-) white dwarfs from the Sloan Digital Sky Survey Data Release 4
}

\author{
S. D. Hügelmeyer ${ }^{1}$, S. Dreizler ${ }^{1}$, D. Homeier ${ }^{1}$, J. Krzesiński ${ }^{2,3}$, K. Werner ${ }^{4}$, A. Nitta ${ }^{5}$, and S. J. Kleinman ${ }^{6}$ \\ 1 Institut für Astrophysik, Georg-August-Universität Göttingen, Friedrich-Hund-Platz 1, 37077 Göttingen, Germany \\ e-mail: shuegelm@astro.physik.uni-goettingen.de \\ 2 Apache Point Observatory, New Mexico State University, 2001 Apache Point Road, PO Box 59, Sunspot, NM 88349, USA \\ 3 Mt. Suhora Observatory, Cracow Pedagogical University, ul. Podchorazych 2, 30-084 Cracow, Poland \\ 4 Institut für Astronomie und Astrophysik, Eberhard-Karls-Universität Tübingen, Sand 1, 72076 Tübingen, Germany \\ 5 Gemini Observatory, 670 N. A’Ohoku Place, Hilo, HI 96720, USA \\ ${ }^{6}$ Subaru Telescope, National Astronomical Observatory of Japan, 650 N. A'Ohoku Place, Hilo, HI 96720, USA
}

Received 17 January 2006 / Accepted 10 April 2006

\begin{abstract}
Context. The Sloan Digital Sky Survey Data Release 4 has provided spectra of several new PG 1159 stars and DO white dwarfs. This increase in known hot H-deficient compact objects significantly improves the statistics and helps to investigate late stages of stellar evolution.

Aims. From the optical SDSS spectra, effective temperatures and surface gravities are derived in order to place the observed objects in an evolutionary context. Especially the connection between PG 1159 stars and DO white dwarfs shall be investigated.

Methods. Using our non-LTE model atmospheres and applying $\chi^{2}$-fitting techniques, we determine stellar parameters and their errors. We derive total stellar masses for the DO white dwarfs using model evolutionary tracks.

Results. We confirm three PG 1159 stars, with one showing ultra-high excitation ion features, and one sdO which we originally classified as a PG 1159 star. Additionally, we re-analysed the known PG 1159 star, PG 1424+535, with our new models. Furthermore, we present the first spectral analyses of thirteen DO white dwarfs, three of which show M-star features in their spectra, while two display ultra-high excitation ion features.
\end{abstract}

Key words. stars: abundances - stars: fundamental parameters - stars: evolution - stars: AGB and post-AGB -

stars: white dwarfs - stars: binaries: spectroscopic

\section{Introduction}

White dwarfs (WDs) represent the final evolutionary stage for over $90 \%$ of all stars (initial mass $M_{\mathrm{i}}<8 M_{\odot}$ ). Due to very high mass loss at the tip of the Asymptotic Giant Branch (AGB), WD progenitors lose part of their envelope, forming a planetary nebula in their subsequent evolution. The remaining core of the star rapidly evolves toward high effective temperatures $\left(T_{\text {eff }}>100000 \mathrm{~K}\right)$. When $\mathrm{H}$ - and He-shell burning cease, the star enters the WD cooling sequence. The evolution of these postAGB objects is separated into a $\mathrm{H}$-rich and $\mathrm{H}$-deficient sequence with a ratio of about $4: 1$.

In their catalog of spectroscopically confirmed white dwarfs from the Sloan Digital Sky Survey (SDSS, York et al. 2000) Data Release 4 (DR4, Adelman-McCarthy et al. 2005), Eisenstein et al. (2006a) report 31 DO white dwarfs and 10 PG 1159 stars. Following our spectral analyses of 16 hot H-deficient objects from the DR4 WD catalog (10 DOs, of which 9 were classified as DOs and one as DB+M, 5 PG 1159 stars, and one sdO; Hügelmeyer et al. 2005) which were included in DR3 (Abazajian et al. 2005), we now extend this work to 13 DOs and 4 PG 1159 stars which are available only since DR4. The remaining 9 DOs from the DR4 WD catalog not analysed in this or our previous paper are either too noisy or insufficiently flux calibrated for a reasonable spectral analysis.

\section{1. $P G 1159$ stars}

PG 1159 stars are evolutionary transition objects between the hottest post-AGB and WD phases. The prototype of this spectroscopic class, PG 1159-035, was found in the Palomar Green (PG) survey (Green et al. 1986). It shows a spectrum without detectable $\mathrm{H}$-absorption lines. It is dominated by $\mathrm{He}$ II and highly ionised carbon and oxygen lines. PG 1159 stars are characterised by a broad absorption trough around $4670 \AA$ A composed of He II $4686 \AA$ and several C IV lines, suggesting high effective temperatures. Spectral analyses yield $T_{\text {eff }}=75000-200000 \mathrm{~K}$ and gravities of $\log g=5.5-8.0$ (Werner et al. 1991; Dreizler et al. 1994; Werner et al. 1996). Of the 28 PG 1159 stars known prior to the SDSS, ten are low-gravity (subtype lgE, Werner 1992) stars, placing them in the same Hertzsprung-RussellDiagram (HRD) region as the hot central stars of planetary nebulae (CSPNe), while the others are more compact objects with surface gravities of WDs (subtype A or E). Due to their rarity, the majority of the known PG 1159 stars were discovered in large surveys (Palomar Green, Hamburg Schmidt (HS), Hagen et al. 1995). The most recent and only discovery within the last 10 years, besides those from the SDSS (Werner et al. 2004), was an object from the Hamburg ESO (HE) survey (Wisotzki et al. 1996). The SDSS thus offers a new and relatively unique opportunity to increase the number of known PG 1159 stars. 
The star PG 1159-035 (=GW Vir) also defines a class of variable stars. McGraw et al. (1979) discovered low-amplitude non-radial $g$-mode pulsations in this object. About one third of the PG 1159 stars show this variability which is thought to be driven by cyclic ionisation of carbon and oxygen as suggested by Cox (1986) and Starrfield (1987). Analyses of HST spectra of spectroscopically identified PG 1159 stars indicate that there is a likely relation between high carbon and oxygen abundances and pulsations (Dreizler \& Heber 1998, hereafter DH98), which is corroborated by theoretical calculations of Quirion et al. (2004).

The region in the HRD occupied by the PG 1159 stars overlaps with that of the DO white dwarfs. Therefore, it is assumed that gravitational settling of the heavier elements in the atmosphere of the PG 1159 stars leads them to transition towards DO white dwarfs.

\subsection{DO white dwarfs}

White dwarfs can be separated into two distinct spectroscopic classes, DA and non-DA white dwarfs. The former show a pure hydrogen spectrum and can be found over the entire WD cooling sequence. The latter fall into three subclasses: DO $(45000 \mathrm{~K}<$ $\left.T_{\text {eff }}<120000 \mathrm{~K}\right)$, DB $\left(11000 \mathrm{~K}<T_{\text {eff }}<30000 \mathrm{~K}\right)$, and $\mathrm{DC} / \mathrm{DQ} / \mathrm{DZ}$ white dwarfs $\left(T_{\mathrm{eff}}<11000 \mathrm{~K}\right.$; pure continuum/carbon/metal lines present). The spectroscopic appearance of the DO and DB subclasses is determined by the ionisation balance of He I and He II. DO white dwarfs show a pure He II spectrum at the hot end and a mixed He I/II spectrum at the cool end. The transition to the cooler DB dwarfs, characterised by pure He I spectra, is interrupted by the so-called "DB gap" (Liebert et al. 1986). In the HRD region of white dwarfs with $30000 \mathrm{~K}<$ $T_{\text {eff }}<45000 \mathrm{~K}$, no objects with H-deficient atmospheres have been observed to date. However, Eisenstein et al. (2006b) describe over 25 objects taken from DR4 data which likely fall in that temperature region. These new objects might increase our knowledge of the spectral evolution of He-rich white dwarfs considerably.

Additional constraints on stellar parameters may result from the analysis of DO white dwarfs in binary systems. Currently, three DOs with late type companions (PG 0046+078, PG 0237+116, and HD 149499 B) are known. The first two have M-star companions with only a minor contribution to the combined spectrum (Heber et al. 1996; Wesemael et al. 1985). The latter one is accompanied by a much brighter K-star which complicates the analysis of the optical spectrum of the DO (Wegner 1979).

As in the case of PG 1159 stars, the most recent discovery of a DO white dwarf comes from the HE survey (Werner et al. 2004), while the PG and HS surveys have contributed the majority of the 19 DOs known prior to the SDSS.

\section{Spectral analysis}

In order to analyse the DO and PG 1159 spectra (SDSS observations for these objects are described in Hügelmeyer et al. 2005), we calculated homogeneous, plane-parallel, non-LTE model atmospheres with a code based on the Accelerated Lambda Iteration method (Werner et al. 2003, and references therein). For these types of stars, it is necessary to account for non-LTE effects in our models, as shown by Dreizler \& Werner (1996) for DO white dwarfs and by Werner et al. (1991) for PG 1159 stars. To compare our synthetic spectra to the observed spectra, we normalise the observed spectra with third order polynomials fit through the continua. The continua are determined by the normalised theoretical spectra. Lineshifts due to radial velocities are taken into account by means of cross-correlation. This comparison procedure is performed by an IDL code routine in order to guarantee consistent results. We used a $\chi^{2}$-statistic to derive best-fit models and computed 1- $\sigma$ errors following Zhang et al. (1986).

\subsection{Spectral analysis of $P G 1159$ stars}

For the PG 1159 stars in our sample, we calculated model atmospheres using detailed $\mathrm{H}-\mathrm{He}-\mathrm{C}-\mathrm{N}-\mathrm{O}$ model atoms. The model grid ranges from $T_{\text {eff }}=55000-150000 \mathrm{~K}$ and $\log g=5.5-7.8$. The abundances are fixed to values $\mathrm{He} / \mathrm{H}=100$ and $\mathrm{C} / \mathrm{He}=$ $0.01,0.03,0.05,0.07,0.09,0.10,0.11,0.20,0.30$, or 0.60 by number. We have a nearly complete model grid with $T_{\text {eff }}=$ $55000-110000 \mathrm{~K}$ in steps of $5000 \mathrm{~K}, \log g=6.4-7.8$ in steps of $0.2 \mathrm{dex}$, and $\mathrm{C} / \mathrm{He}=0.01-0.11$ in steps of 0.02 . Complete coverage of the whole parameter space is not yet available due to the high computational time required to compute the model atmospheres. However, the majority of the analysed PG 1159 stars are covered by our nearly complete model grid. Best-fit models for PG 1159 star candidates were calculated with an oxygen abundance following the typical PG 1159 abundance-scaling ratio $\mathrm{O} / \mathrm{C} \approx \mathrm{C} / \mathrm{He}$. However, variations in the oxygen abundance do not produce a significant effect on the other stellar parameters. The nitrogen abundance is kept fixed at $\mathrm{N} / \mathrm{He}=0.01$ by number which is a typical upper limit for PG 1159 stars. The analysed spectra of PG 1159 stars and their best-fit models are shown in Fig. 1.

\subsection{Spectral analysis of DO white dwarfs}

Detailed H-He atomic models (Dreizler \& Werner 1996) were used to calculate our model atmospheres. The model grid ranges from $T_{\text {eff }}=40000-120000 \mathrm{~K}$ in steps of $2500 \mathrm{~K}, \log g$ ranges from 7.0 to 8.4 in intervals of 0.2 dex. The helium abundance is fixed to $\mathrm{He} / \mathrm{H}=99$. The spectra of our analysed DOs, together with their corresponding best-fit models, are shown in Fig. 2.

\subsection{Analysis of spectra showing DO and M dwarf features}

In order to derive stellar parameters for both stars from the DO+M star spectra, we used the DO model atmospheres previously described in Sect. 2.2 along with theoretical LTE M-star atmospheres calculated with the PHOENIX code (Hauschildt et al. 1999). Their construction follows the setup used for the preliminary GAIA grid (Brott \& Hauschildt 2005), but has been updated to use PHOENIX version 14.1 and compute spectra at $0.2 \AA$ resolution. Major updates to the input physics include the adaption of the revised solar composition of Asplund et al. (2005) for the chemical equilibrium abundances and a correction to the partition sum for TiO, leading to $\sim 3$-times greater line strengths for this important absorber (Allard et al., in prep.).

For the fit of both spectral components, a Planck spectrum was first fitted to represent the Rayleigh-Jeans tail contribution of the DO in the red part of the spectrum; then we selected a model spectrum based on an initial fit of the spectral type of the cool companion and subtracted it from the observation. Next, we used our $\chi^{2}$-fitting routines to determine the best DO fit. This model was then subtracted from the combined observed spectrum and the remaining cool star spectrum was fitted to a grid of models spanning $T_{\text {eff }}=2600-5000 \mathrm{~K}$ in steps of $100 \mathrm{~K}$ and $\log g=3.5-5.5$ in steps of $0.5 \mathrm{dex}$. 


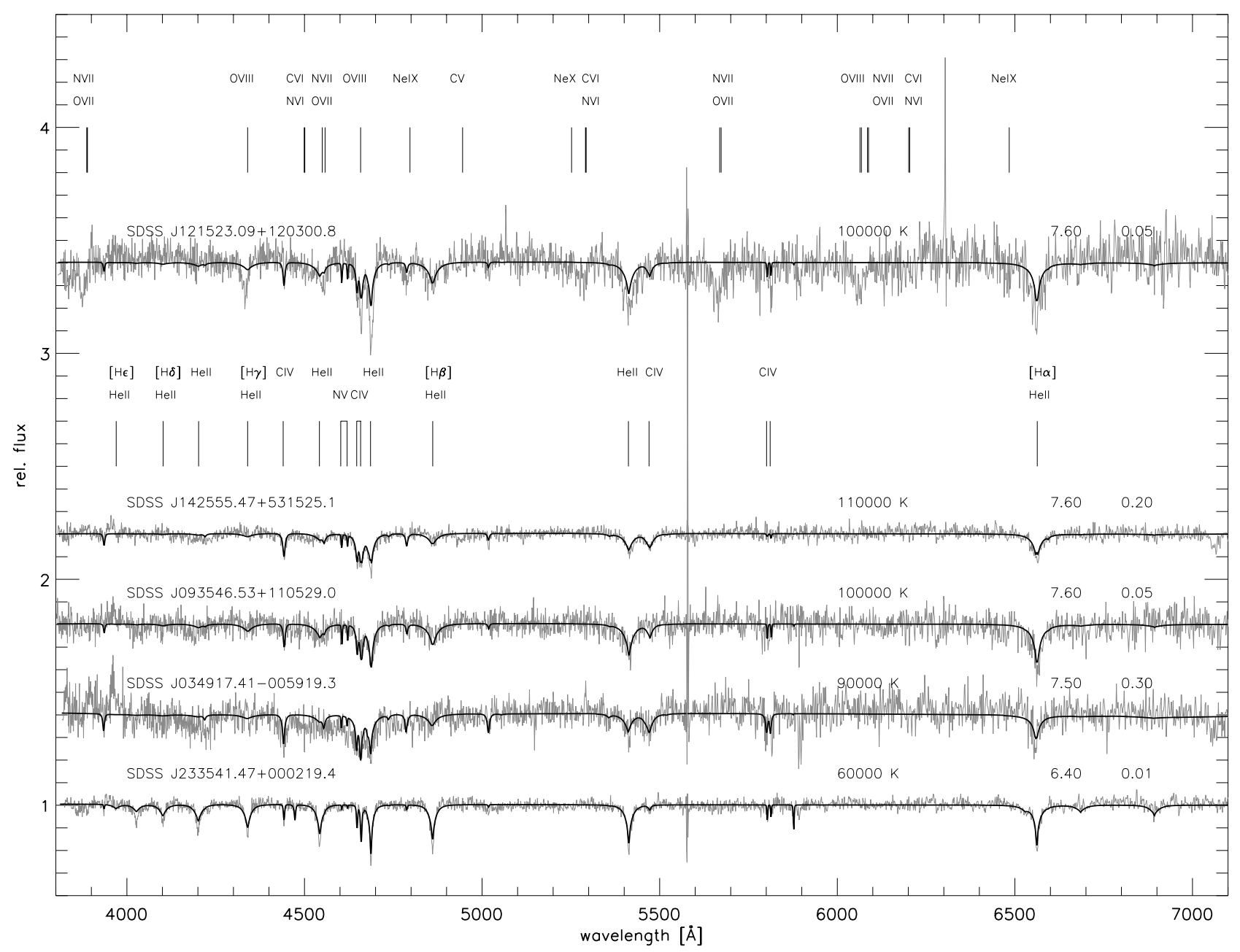

Fig. 1. Normalised optical spectra (grey lines) of PG 1159 stars and the sdO star (bottom spectrum) along with model atmosphere fits (black lines). The top object shows ultra-high excitation ion features. Object names are printed on the left, with effective temperatures, logarithmic surface gravities, and carbon abundances $(\mathrm{C} / \mathrm{He})$, on the right.

The best fit was determined by $\chi^{2}$-minimisation over the entire spectral range; however we assigned a reduced weight to the regions from $6430-6560 \AA$ and $6720-6950 \AA$. In this part of the $\mathrm{TiO}$ bands, the models reproduce the observations rather poorly, as has been noted in previous analyses of $\mathrm{M}$ dwarfs (e.g. Leggett et al. 2002). A reason for this mismatch is probably superposed absorption due to $\mathrm{CaH}$, for which no line opacity data are available in the PHOENIX version used for these models. Despite these shortcomings we find acceptable fits for SDSS J091621.83+052119.2 and SDSS J133633.22-013116.5 at $3600-3700 \mathrm{~K}$, with the fit quality rapidly degrading towards higher and lower $T_{\text {eff }}$. In light of the unknown systematic errors due to the incomplete molecular data, flux calibration errors, and possible interstellar reddening, we conservatively estimate an uncertainty in $T_{\text {eff }}$ of $100 \mathrm{~K}$. The much fainter spectrum of the companion to SDSS J075606.36+421613.0 is consistent with temperatures from at least $3000-3600 \mathrm{~K}$. In all cases, the spectra show very little sensitivity to gravity within a range of $4.0<\log g<5.5$. A direct determination of the stellar radius is therefore not possible from these data, but the fits are entirely consistent with the assumption of main sequence stars and the companion radius can therefore be determined from a standard mass-radius-relation as well as from evolutionary tracks. We did not find any improvement of the fits using metallicities either higher or lower than solar.
Finally, we subtracted the best-fit M-star model atmosphere from the observed spectrum and used our standard $\chi^{2}$-fitting routines again to refine $T_{\text {eff }}$ and $\log g$ for the DO white dwarf. Only minor differences in effective temperatures and surface gravities compared to the first DO model fit were derived. The three spectra showing DO and M-star features, together with combined DO and M-star models, are presented in Fig. 6.

\section{Results and discussion}

After presenting ten DO white dwarfs and five PG 1159 stars in Hügelmeyer et al. (2005), we now extend our spectral analyses to the recently published SDSS DR4 DOs and PG 1159 stars. We present results from this work and Hügelmeyer et al. (2005) together in Tables 1 and 2. Some atmospheric parameters for the PG 1159 stars analysed in Hügelmeyer et al. (2005) have changed after applying our $\chi^{2}$-fitting routines to these objects. The statistical errors provided by the $\chi^{2}$-analysis and presented in Tables 1 and 2 are very small, while suspected systematic errors are of the order of the grid steps described in Sect. 2.

\section{1. $P G 1159$ stars}

We found six spectra from the SDSS DR4 WD catalog (Eisenstein et al. 2006a) which we identified as PG 1159 stars, 


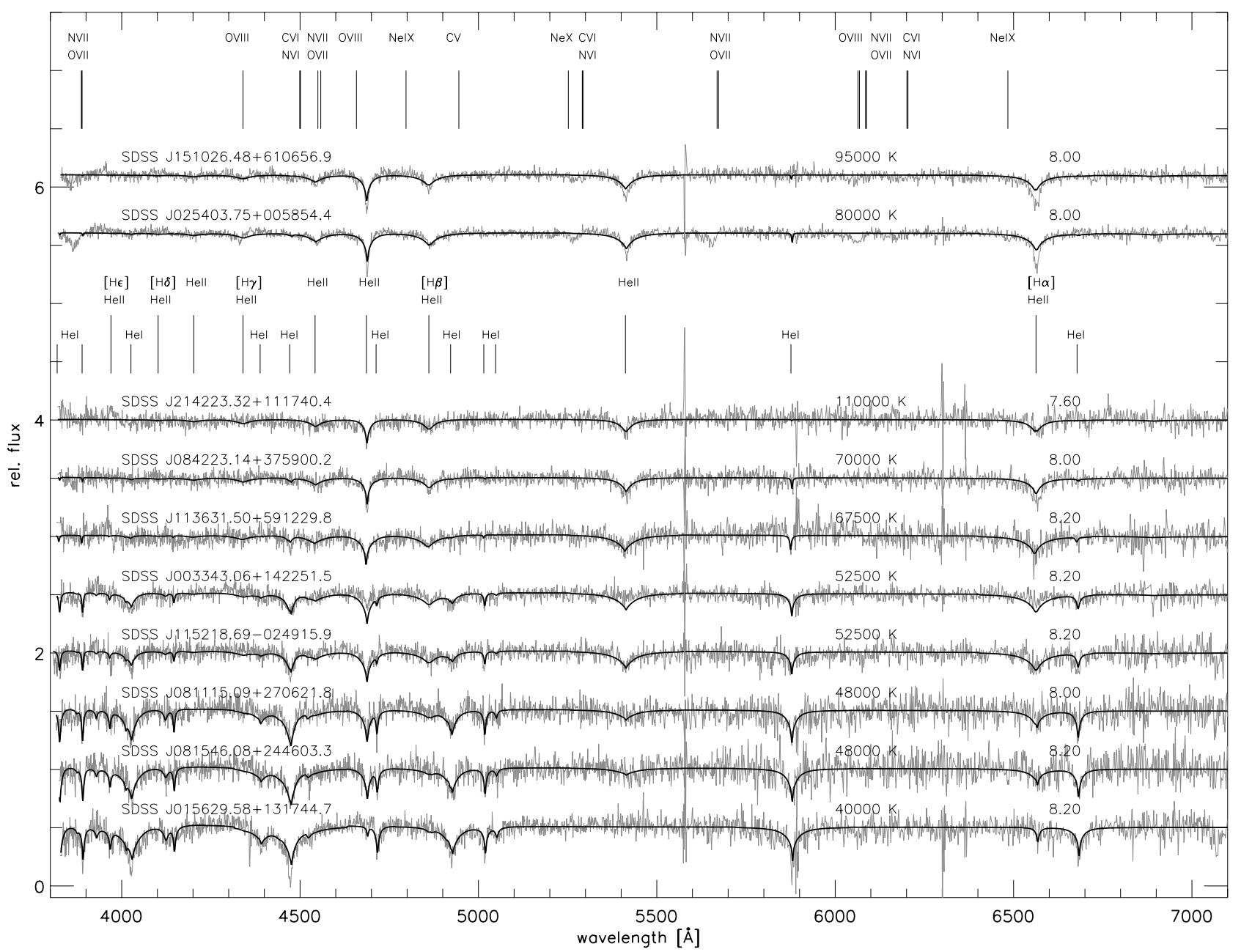

Fig. 2. Normalised optical spectra (grey lines) of DO white dwarfs along with model atmosphere fits (black lines). The two top objects show ultrahigh excitation ion features. Object names are printed on the left, effective temperatures and logarithmic surface gravities on the right. The spectra of the hottest stars are dominated by He II absorption lines, while increasing line strengths of He I are observed with decreasing temperature.

although one (SDSS J233541.47+000219.4) turns out to be best fit as a sdO rather than a PG 1159 star, even though strong $\mathrm{C}$ IV lines are present. Our model grid is not adopted for the analysis of sdO stars and the results are therefore preliminary only. We note that the He II line cores are not well matched with the current best-fit model. This object, known as WD 2333-002, has been previously classified by Liebert et al. (2005) as a $\mathrm{sdB} / \mathrm{sdOB}$.

One of our six selected PG 1159 stars, SDSS $\mathrm{J} 114635.23+001233.5$, was previously known as PG 1144+005. Since no new model atmospheres for this hot object are available, the photospheric parameters $T_{\mathrm{eff}}=150000 \mathrm{~K}$, $\log g=6.50$, and $\mathrm{C} / \mathrm{He}=0.50$ derived by Werner \& Heber (1991) still hold and the spectrum is not analysed in this work.

SDSS J121523.09+120300.8 shows features of ultra-high excitation ions (uhei) and is the first PG 1159 star of that type discovered so far. Lines of O VII and N VII around $3888 \AA$, at $5673 \AA$, and around $6086 \AA$, as well as O VIII at $4340 \AA$, $4658 \AA$, and $6064 / 6068 \AA$ are clearly visible in the spectrum (see Fig. 1). Absorption features of C VI, N VI, Ne IX, and Ne X described by Dreizler et al. (1995), Werner et al. (1995), and Krzesiński et al. (2004) for DO white dwarfs, are also indicated in Fig. 1 and are only ambiguously identifiable due to low signalto-noise and spectral resolution. The observed He II absorption lines are too deep to be fitted with any model. Due to the lack of appropriate modelling of the uhei phenomenon, stellar parameters are derived for this star from a best-fit model which is determined without considering the uhei lines. It should be kept in mind that significantly higher temperatures $\left(T_{\mathrm{eff}}>500000 \mathrm{~K}\right)$ are necessary to excite the uhei lines visible in this spectrum (Dreizler et al. 1995).

The remaining three PG 1159 star candidates are A subtype PG 1159 stars. SDSS J142555.47+531525.1 was already known as PG $1424+535$ or WD $1424+535$ and has been analysed by DH98 and Werner et al. (1991) who derived $T_{\text {eff }}=$ $110000 \mathrm{~K} / 100000 \mathrm{~K}$, respectively, $\log g=7.00$, and $\mathrm{C} / \mathrm{He}=$ 0.30 using UV and optical HST spectra. Our fit provides the same effective temperature as DH98, but a higher surface gravity $(\log g=7.60)$, and a lower carbon abundance $(\mathrm{C} / \mathrm{He}=0.20)$ for the new SDSS spectrum of this object. In our analysis we also fit the spectrum with the model used by DH98, but we obtained a slightly lower $\chi^{2}$-value for our new model. However, the models used are not very sensitive to carbon abundance changes above $\mathrm{C} / \mathrm{He} \approx 0.10$ in the optical.

The stellar parameters resulting from these spectral analyses are listed in Table 1. We have also included results from time-resolved photometric observations of four candidates for pulsating PG 1159 stars in Table 1, obtained at the Calar Alto Observatory $2.2 \mathrm{~m}$ telescope in March 2005. In Fig. 3, positions of the four analysed PG 1159 stars and the one sdO star are 
Table 1. Photospheric parameters of our PG 1159 star sample. The C/He abundance ratio is given by number. Objects marked with an asterisk $\left({ }^{*}\right)$ have been analysed in Hügelmeyer et al. (2005). The PG 1159 stars which have been observed for pulsations do not show variations above the specified detection limit $\sigma$.

\begin{tabular}{lcccl}
\hline \hline $\begin{array}{l}\text { Name } \\
\text { SDSS J }\end{array}$ & $\begin{array}{c}T_{\text {eff }} \\
{[\mathrm{kK}]}\end{array}$ & $\begin{array}{c}\log g \\
{[\mathrm{cgs}]}\end{array}$ & $\mathrm{C} / \mathrm{He}$ & remarks \\
\hline $001651.42-011329.3^{*}$ & $120.0 \pm 2.3$ & $5.50 \pm 0.49$ & $0.30 \pm 0.006$ & \\
$102327.41+535258.7^{*}$ & $110.0 \pm 1.6$ & $7.60 \pm 0.03$ & $0.20 \pm 0.007$ & non-variable $(\sigma=12 \mathrm{mmag})$ \\
$142555.47+531525.1$ & $110.0 \pm 0.9$ & $7.60 \pm 0.01$ & $0.20 \pm 0.004$ & known as PG 1424+535 $(\mathrm{DH} 98)$ \\
$075540.94+400918.0^{*}$ & $100.0 \pm 2.6$ & $7.60 \pm 0.05$ & $0.03 \pm 0.006$ & \\
$093546.53+110529.0$ & $100.0 \pm 1.3$ & $7.60 \pm 0.03$ & $0.05 \pm 0.002$ & non-variable $(\sigma=15 \mathrm{mmag})$ \\
$121523.09+120300.8$ & $100.0 \pm 0.8$ & $7.60 \pm 0.02$ & $0.05 \pm 0.002$ & shows ultra-high excitation ion absorption \\
$134341.88+670154.5^{*}$ & $100.0 \pm 0.7$ & $7.60 \pm 0.02$ & $0.05 \pm 0.001$ & non-variable $(\sigma=9$ mmag) \\
$144734.12+572053.1^{*}$ & $100.0 \pm 1.6$ & $7.60 \pm 0.02$ & $0.05 \pm 0.002$ & non-variable $(\sigma=10$ mmag $)$ \\
$034917.41-005919.3$ & $90.0 \pm 0.9$ & $7.50 \pm 0.01$ & $0.30 \pm 0.019$ & \\
\hline $233541.47+000219.4$ & $60.0 \pm 0.9$ & $6.40 \pm 0.01$ & $0.01 \pm 0.001$ & $(\mathrm{sdO})$ known as WD 2333-002 \\
$110215.46+024034.2^{*}$ & $55.0 \pm 0.8$ & $6.40 \pm 0.02$ & $0.01 \pm 0.003$ & $(\mathrm{sdO})$ \\
\hline
\end{tabular}

Table 2. Stellar parameters of our SDSS DO white dwarf sample. The three objects below the line show spectra with DO and M-star features. The letters A and B denote the DO and the M-star component, respectively. Objects marked with an asterisk $\left(^{*}\right)$ have been previously analysed in Hügelmeyer et al. (2005).

\begin{tabular}{lrcc}
\hline \hline Name & \multicolumn{1}{c}{$\begin{array}{c}T_{\text {eff }} \\
\text { SDSS J }\end{array}$} & $\begin{array}{c}\log g \\
{[\mathrm{cgs}]}\end{array}$ & $\begin{array}{c}M \\
{\left[M_{\odot}\right]}\end{array}$ \\
\hline $091433.61+581238.1^{*}$ & $120.0 \pm 1.7$ & $8.00 \pm 0.02$ & 0.75 \\
$204158.98+000325.4^{*}$ & $110.0 \pm 4.5$ & $7.20 \pm 0.04$ & 0.60 \\
$214223.32+111740.4$ & $110.0 \pm 2.4$ & $7.60 \pm 0.05$ & 0.60 \\
$154752.33+423210.9^{*}$ & $100.0 \pm 1.6$ & $7.60 \pm 0.06$ & 0.59 \\
$151026.48+610656.9^{1}$ & $95.0 \pm 0.8$ & $8.00 \pm 0.04$ & 0.71 \\
$084008.72+325114.6^{*}$ & $85.0 \pm 1.1$ & $8.40 \pm 0.08$ & 0.90 \\
$025403.75+005854.4^{1}$ & $80.0 \pm 0.5$ & $8.00 \pm 0.06$ & 0.68 \\
$155356.81+483228.6^{*}$ & $75.0 \pm 1.2$ & $8.00 \pm 0.09$ & 0.68 \\
$084223.14+375900.2$ & $70.0 \pm 0.3$ & $8.00 \pm 0.06$ & 0.68 \\
$140409.96+045739.9^{*}$ & $70.0 \pm 0.5$ & $8.00 \pm 0.04$ & 0.68 \\
$113631.50+591229.8$ & $67.5 \pm 1.6$ & $8.20 \pm 0.13$ & 0.78 \\
$131724.75+000237.4^{*, 2}$ & $62.5 \pm 0.5$ & $7.80 \pm 0.03$ & 0.58 \\
$034101.39+005353.0^{*}$ & $55.0 \pm 0.4$ & $8.00 \pm 0.05$ & 0.65 \\
$003343.06+142251.5$ & $52.5 \pm 0.2$ & $8.20 \pm 0.01$ & 0.76 \\
$115218.69-024915.9$ & $52.5 \pm 0.3$ & $8.20 \pm 0.02$ & 0.76 \\
$034227.62-072213.2^{*}$ & $50.0 \pm 0.2$ & $8.00 \pm 0.03$ & 0.65 \\
$081115.09+270621.8$ & $48.0 \pm 0.3$ & $8.00 \pm 0.04$ & 0.64 \\
$113609.59+484318.9^{*}, 3$ & $48.0 \pm 0.1$ & $8.00 \pm 0.04$ & 0.64 \\
$081546.08+244603.3$ & $48.0 \pm 0.2$ & $8.20 \pm 0.01$ & 0.76 \\
$015629.58+131744.7$ & $40.0 \pm 0.2$ & $8.20 \pm 0.01$ & 0.75 \\
\hline $091621.83+052119.2(\mathrm{~A})$ & $60.0 \pm 5.0$ & $8.00 \pm 0.20$ & 0.66 \\
$091621.83+052119.2$ (B) & $3.7 \pm 0.1$ & $4.75 \pm 0.50$ & 0.51 \\
$133633.22-013116.5 \mathrm{~A}$ & $52.5 \pm 0.5$ & $7.60 \pm 0.06$ & 0.48 \\
$133633.22-013116.5 \mathrm{~B}$ & $3.6 \pm 0.1$ & $4.75 \pm 0.50$ & 0.45 \\
$075606.36+421613.0 \mathrm{~A}$ & $52.5 \pm 0.8$ & $7.40 \pm 0.05$ & 0.42 \\
$075606.36+421613.0 \mathrm{~B}$ & $3.2 \pm 0.2$ & $5.00 \pm 0.50$ & 0.18 \\
\hline
\end{tabular}

1 Shows ultra-high excitation ion absorption; ${ }^{2}$ previously known as HE $1314+0018$ (Werner et al. 2004); ${ }^{3}$ previously known as WD 1133+489 (Dreizler et al. 1997).

compared with evolutionary tracks as well as positions of previously known PG 1159 stars including those from DR3 (Hügelmeyer et al. 2005). The typical SDSS PG 1159 star has approximately $T_{\text {eff }}=100000 \mathrm{~K}, \log g=7.5$, and $\mathrm{C} / \mathrm{He}=$ $0.05-0.30$.

The position of SDSS J233541.47+000219.4 favours our classification of it as a sdO star. It fits the evolutionary tracks of Dorman et al. (1993) within the tiny error bars very well and therefore suggests an Extended Horizontal Branch rather than an AGB history.

\subsection{DO white dwarfs}

Stellar parameters for 13 previously unanalysed DO white dwarfs are presented in Table 2. Three objects show M-star features in their spectra and two display uhei lines (classified by Krzesiński et al. 2004). Table 3 lists the DO white dwarfs from the DR4 WD catalog not analysed here - mainly because their spectra are too noisy.

The two uhei DO white dwarfs show absorption lines of O VII and N VII around $3888 \AA$, at $5673 \AA$, and around $6086 \AA$, as well as O VIII at $4340 \AA, 4658 \AA$, and $6064 / 6068 \AA$. Ne IX signatures are also present in SDSS J025403.75+005854.4. The He II lines, especially the one at $6556 \AA$, are very strong and cannot be fitted by our models. As mentioned above, the effective temperature required to excite these ions to a degree as detected in the two uhei DOs exceeds $500000 \mathrm{~K}$. A massive post-AGB remnant of $1.2 M_{\odot}$ can reach effective temperatures up to $700000 \mathrm{~K}$ on very short time scales (Paczyński 1970), yet, Werner et al. (1995) argue that uhei features in DO white dwarfs cannot be of photospheric origin since He II lines would disappear completely at such high temperatures. The hypothesis of optically thick and hot stellar winds was proposed by the authors due to the triangular shape of the line profile. Furthermore, the lines are blue shifted, which favours the assumption of an expanding envelope. Neglecting this very hot component in the spectral analyses, the uncertainties given by the $\chi^{2}$-fitting procedure are likely to be lower than the systematic errors due to incomplete modelling of the uhei phenomenon.

SDSS J003343.06+142251.5 and SDSS J015629.58+ 131744.7 have been classified by Krzesiński et al. (2004) as DBAO and DBO, respectively, while the former is listed as DO and the latter as DBO in the DR4 WD catalog. Comparing the spectrum of the former object to our DO models, we note that He II lines at $4686 \AA, 5412 \AA$, and $6563 \AA$ are stronger in the model than in the observation. He I is well fitted in the whole spectrum. In contrast, He I lines at $4026 \AA$ and $4471 \AA$ are stronger in the observation than in the model for the latter. The extension of the model grid to even lower model temperatures does not improve the fit of the He I lines since they are almost saturated. The rest of the He I lines in the model and the observation match. SDSS J015629.58+131744.7 appears to be the coolest DO known to date. The addition of small amounts of hydrogen did not lead to a better fit for SDSS J003343.06+142251.5, so that we cannot explain the weak He II lines by traces of hydrogen in the atmosphere. 


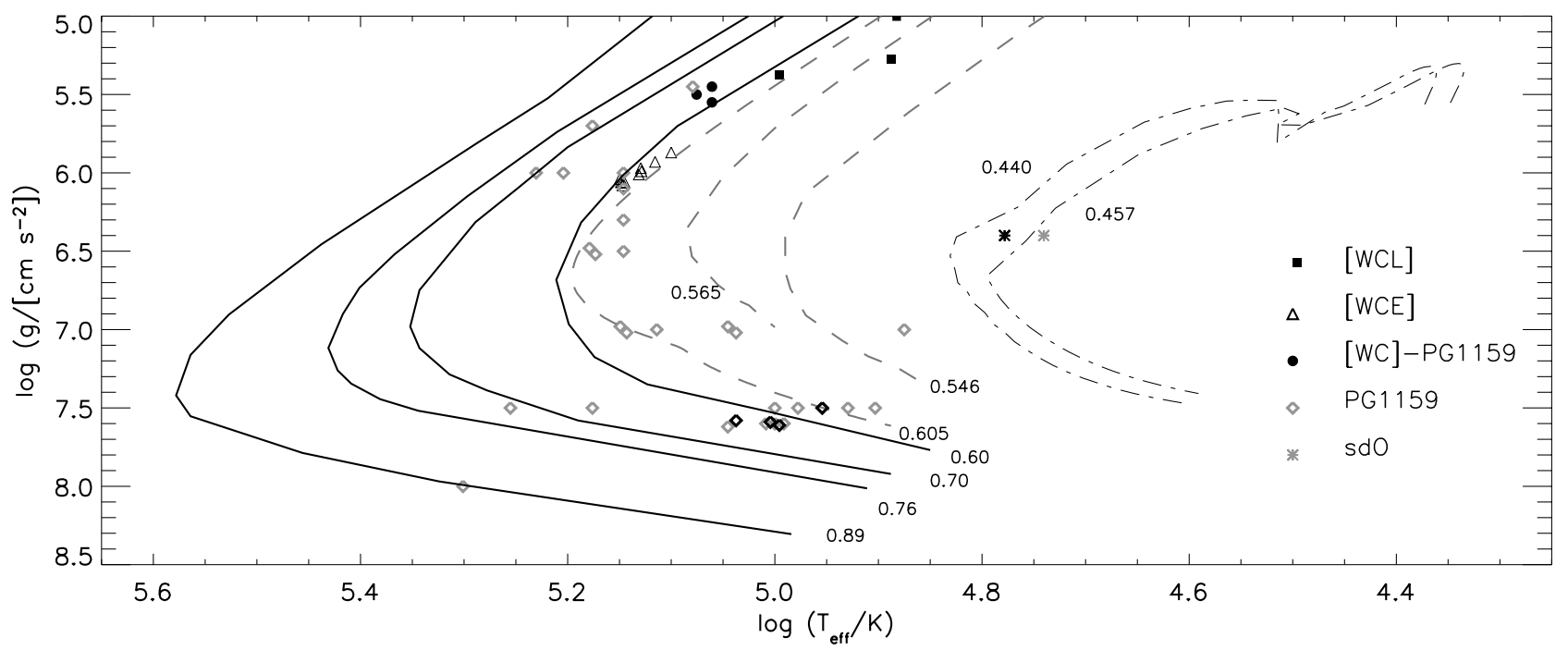

Fig. 3. Positions of the PG 1159 stars (black diamonds) analysed in this paper compared to evolutionary tracks from Blöcker (1995) and Schönberner (1983) (dashed lines), and Wood \& Faulkner (1986) (solid lines). The sdO star from this work (black asterisk) and the one from Hügelmeyer et al. (2005) are plotted together with tracks from Dorman et al. (1993) (dash-dotted lines). Statistical error bars for all analysed objects are smaller than the symbols. The plot also includes previously known PG 1159 stars and proposed progenitor objects (early and late Wolf-Rayet CSPNe:[WCE], [WCL], and [WC]-PG 1159). Labels: mass in $M_{\odot}$.

Table 3. DO white dwarfs from the DR4 WD catalog not analysed in this work due to noisy spectra or as denoted.

\begin{tabular}{ll}
\hline \hline Name & Name \\
SDSS J & SDSS J \\
\hline $011346.78+002828.7^{1,2}$ & $140615.80+562725.9$ \\
$074538.17+312205.3$ & $150053.99+025143.2$ \\
$081533.08+264646.4$ & $205930.25-052848.9^{3}$ \\
$083504.76+090111.7$ & $213932.48+112611.2$ \\
$130248.99-013309.5$ & \\
\hline
\end{tabular}

${ }^{1}$ Insufficiently flux calibrated; ${ }^{2}$ previously known as HS $0111+0012$ (Dreizler \& Werner 1996); ${ }^{3}$ dent in spectrum at $\sim 5800 \AA$.

Masses for all objects are derived from our photospheric parameters and evolutionary tracks from Wood (1995). The thirteen analysed DOs are plotted with these tracks in Fig. 5 and the mass distribution for all DO stars is shown in Fig. 4. As already mentioned in Hügelmeyer et al. (2005), DOs from the SDSS seem to have higher masses than those analysed before the SDSS. We assume that this is an effect introduced by the application of our automated fitting routines which favour good line wing fits, thus steering the fits towards higher surface gravities.

\subsection{Combined DO and $M$ dwarf spectra}

The three observed spectra that show both DO and M-star features, along with combined DO and M-star model atmospheres are shown in Fig. 6. The flux of each model is multiplied by the squared radius of the individual component. The radii are derived from the surface gravity and mass which come from evolutionary tracks from Wood (1995) for the DOs and Baraffe et al. (1998) for the M-stars. A constant factor which accounts for our distance from the source is multiplied to DO and M-star model fluxes. The total flux clearly fits SDSS J133633.22-013116.5 and SDSS J075606.36+421613.0 within the uncertainties, suggesting that the spectra come from binary systems. SDSS J091621.83+052119.2, however, shows a deviation between combined models and observation which cannot

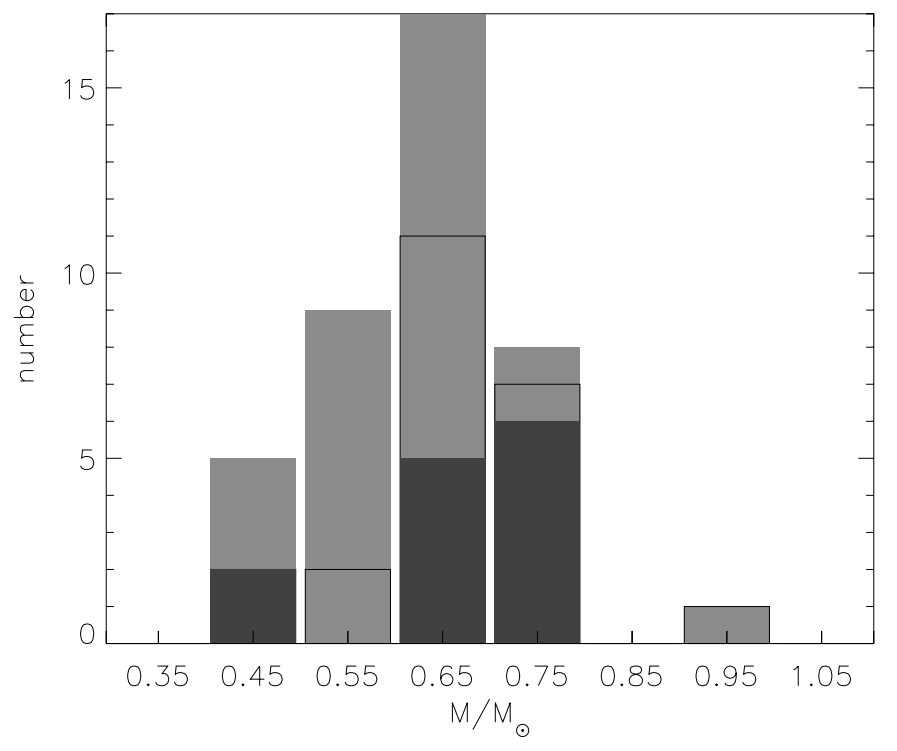

Fig. 4. Mass distribution $\left(\right.$ bin $=0.1 M_{\odot}$ ) of all known DO white dwarfs. The black bars represent masses of stars from this work while the area under the black lines shows results from all the SDSS spectral analyses (Hügelmeyer et al. 2005; and this work).

be explained by the uncertainties in the stellar parameters. We therefore propose that the spectrum originates from two unrelated stars on the same line of sight. This is also corroborated by the images of the objects, which have ellipsoidal shapes and different photometric centres in different colors ${ }^{1}$. A sum of DO and M-star models scaled individually for distances fit the observation well (dashed line in Fig. 6) showing that the model parameters were derived properly.

Stellar parameters for the M-star components are shown in Table 2. Our fitting routines found a best-fit model with $T_{\text {eff }}=55000 \mathrm{~K}$ for the DO part of SDSS J091621.83+052119.2

\footnotetext{
1 See http://cas.sdss.org/dr4/en/tools/explore/ obj . asp?id=587732578300657729
} 


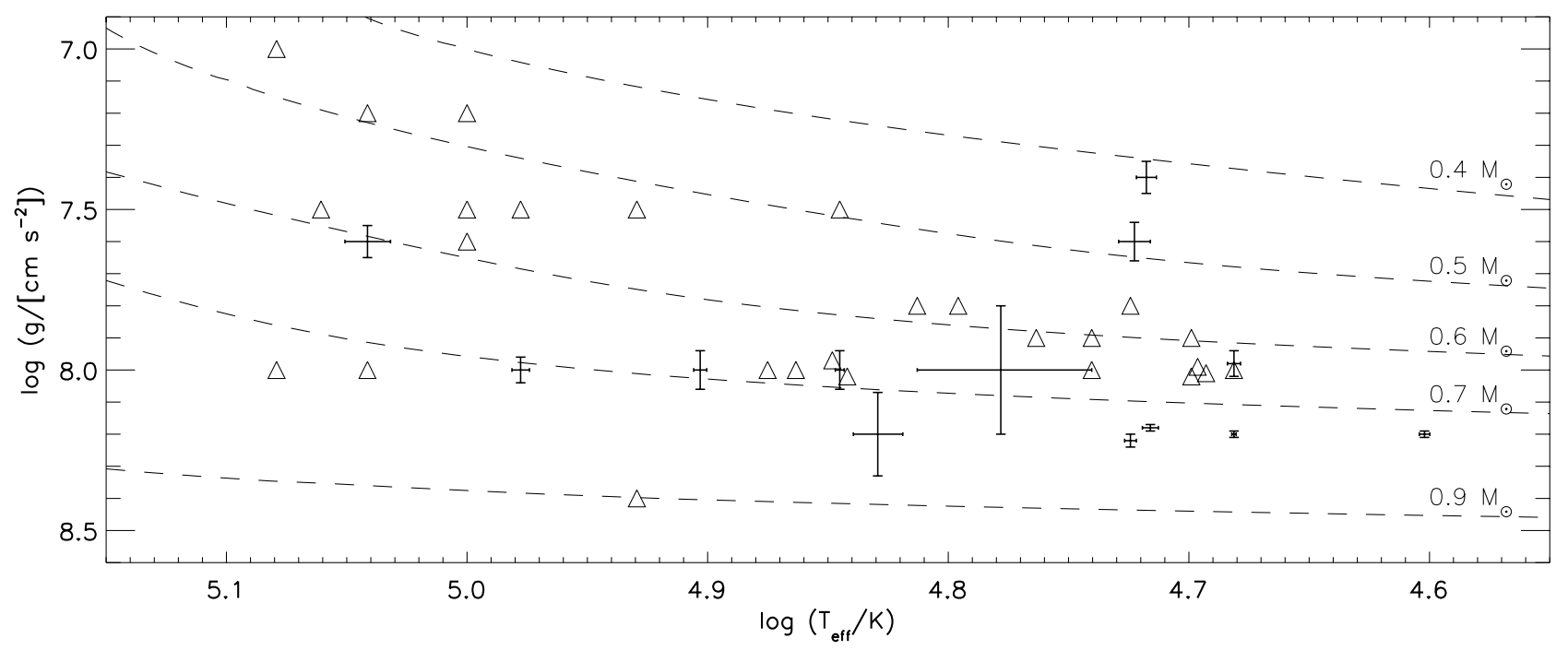

Fig. 5. Positions of DO white dwarfs compared with evolutionary tracks from Wood (1995). The triangles represent the 27 hitherto known DOs (see Dreizler \& Werner 1996; Dreizler et al. 1997; Werner et al. 2004; Hügelmeyer et al. 2005).

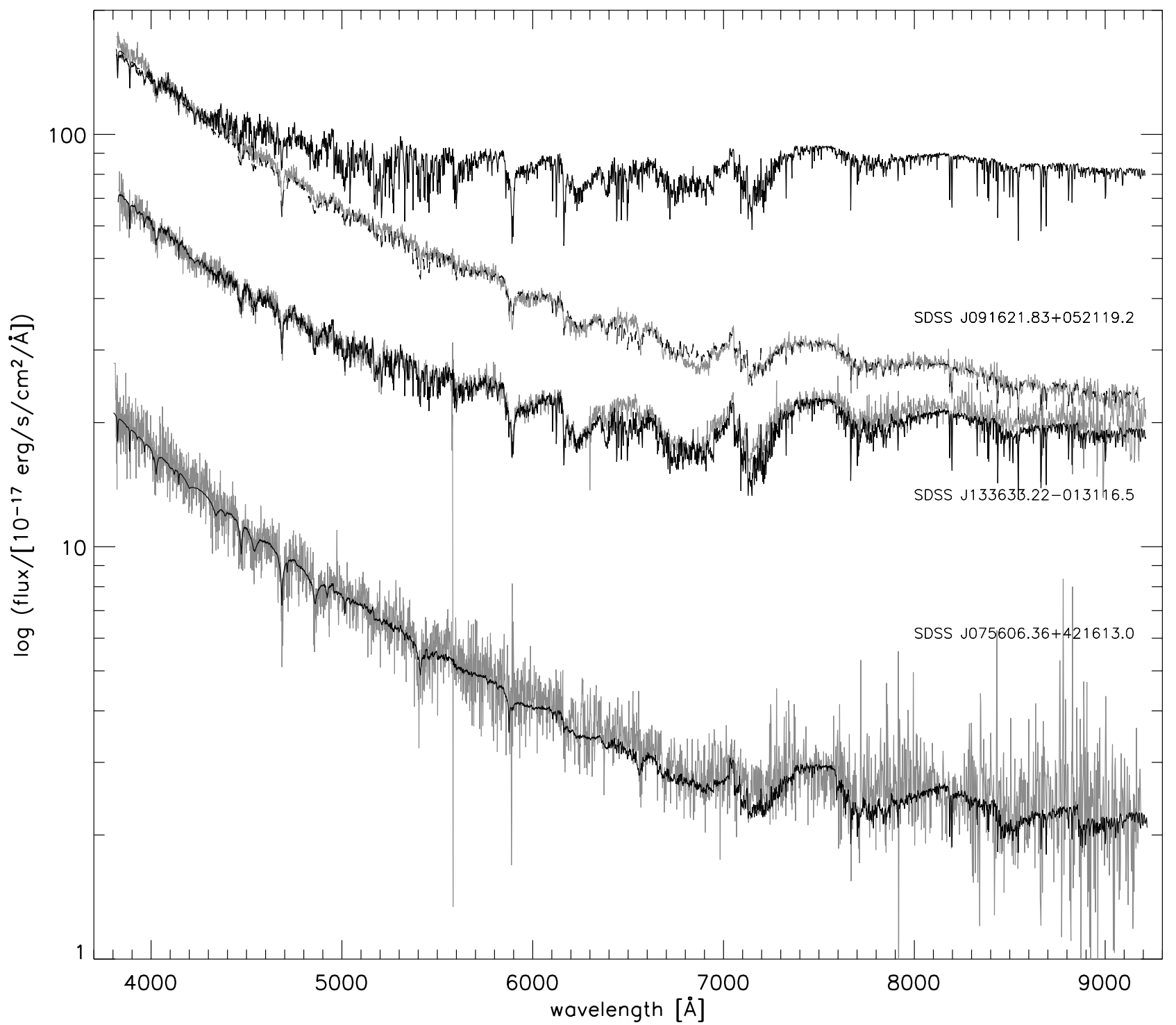

Fig. 6. Observed spectra of two DO+M binaries (bottom two grey lines) and of one DO white dwarf and M-star pair which are coincidentally in the same line of sight. Combined model atmospheres for the two components are shown as black lines. The black dashed line plotted over SDSS J091621.83+052119.1 is the combination of the DO and M-star models where scaling is performed for different distances. 
using the normalised spectrum. However, the flux distribution of the observed spectrum favours an effective temperature of $T_{\text {eff }}=60000 \mathrm{~K}$. We chose the latter value since M-star contributions in the normalised spectrum (observation minus M-star model) were still significant. Error estimates are 0.2 dex for $\log g$ and $5000 \mathrm{~K}$ in $T_{\text {eff }}$ because the continuum distribution is not properly fitted with parameters which exceed the range given by these errors.

\section{Summary}

We have performed spectral analyses of 18 hot H-deficient (pre-) white dwarfs that are new in Data Release 4 of the Sloan Digital Sky Survey using our $\chi^{2}$-fitting routines. We confirm three PG 1159 stars, one of which shows lines of ultra-high excitation ions (uhei). A re-analysis of PG $1424+535$ with our $\chi^{2}$-programs using a SDSS spectrum has provided a lower carbon abundance and a higher surface gravity than found by Dreizler \& Heber (1998). Furthermore, thirteen DO white dwarfs have been analysed, two of which show uhei absorption and three of which have M-star features in their spectra. We propose that two of the three latter objects are binary systems. The mass distribution of the DO white dwarfs from the SDSS is shifted towards higher masses compared to the one constructed from analyses before the SDSS.

Acknowledgements. We thank Daniel J. Eisenstein for helpful discussions and comments on the DR4 WD catalog.

We would also like to thank F. Allard for instructive comments on molecular opacities and helpful discussions. Model atmospheres used for this work were calculated in part on the IBM pSeries of the Gesellschaft für wissenschaftliche Datenverarbeitung mbH Göttingen (GWDG).

Funding for the SDSS and SDSS-II has been provided by the Alfred P. Sloan Foundation, the Participating Institutions, the National Science Foundation, the US Department of Energy, the National Aeronautics and Space Administration, the Japanese Monbukagakusho, the Max Planck Society, and the Higher Education Funding Council for England. The SDSS Web Site is http://www. sdss.org/.

The SDSS is managed by the Astrophysical Research Consortium for the Participating Institutions. The Participating Institutions are the American Museum of Natural History, Astrophysical Institute Potsdam, University of Basel, Cambridge University, Case Western Reserve University, University of Chicago, Drexel University, Fermilab, the Institute for Advanced Study, the Japan Participation Group, Johns Hopkins University, the Joint Institute for Nuclear Astrophysics, the Kavli Institute for Particle Astrophysics and Cosmology, the Korean Scientist Group, the Chinese Academy of Sciences (LAMOST), Los Alamos National Laboratory, the Max-Planck-Institute for Astronomy (MPA), the Max-Planck-Institute for Astrophysics (MPIA), New Mexico State University, Ohio State University, University of Pittsburgh, University of Portsmouth, Princeton University, the United States Naval Observatory, and the University of Washington.

Photometric data for the search for pulsations in PG 1159 stars are based on observations collected at the Centro Astronómico Hispano Alemán (CAHA) at Calar Alto, operated jointly by the Max-Planck-Institut für Astronomie and the Instituto de Astrofísica de Andalucía (CSIC).

\section{References}

Abazajian, K., Adelman-McCarthy, J. K., Agüieros, M. A., et al. 2005, AJ, 129, 1755

Adelman-McCarthy, J. K., Agüeros, M. A., Allam, S. S., et al. 2005, ApJS, accepted

Asplund, M., Grevesse, N., \& Sauval, A. J. 2005, in Cosmic Abundances as Records of Stellar Evolution and Nucleosynthesis, ASP Conf. Ser., 336, 25

Baraffe, I., Chabrier, G., Allard, F., \& Hauschildt, P. H. 1998, A\&A, 337, 403 Blöcker, T. 1995, A\&A, 299, 755

Brott, I., \& Hauschildt, P. H. 2005, in The Three-Dimensional Universe with Gaia, ESA SP-576, 565

Cox, A. N. 1986, Highlights Astron., 7, 229

Dorman, B., Rood, R. T., \& O’Connell, R. W. 1993, ApJ, 419, 596

Dreizler, S., \& Werner, K. 1996, A\&A, 314, 217

Dreizler, S., \& Heber, U. 1998, A\&A, 334, 618

Dreizler, S., Werner, K., Jordan, S., \& Hagen, H. 1994, A\&A, 286, 463

Dreizler, S., Heber, U., Napiwotzki, R., \& Hagen, H. J. 1995, A\&A, 303, L53

Dreizler, S., Werner, K., Heber, U., Reid, N., \& Hagen, H. 1997, in The Third Conference on Faint Blue Stars, ed. A. G. D. Philip, J. Liebert, R. Saffer, \& D. S. Hayes (Schenectady, New York: L. Davis Press), 303

Eisenstein, D. J., Liebert, J., Harris, H. C., et al. 2006a, ApJS, submitted Eisenstein, D. J., Liebert, J., Koester, D., et al. 2006b, AJ, submitted

Green, R. F., Schmidt, M., \& Liebert, J. 1986, ApJS, 61, 305

Hagen, H.-J., Groote, D., Engels, D., \& Reimers, D. 1995, A\&AS, 111, 195

Hauschildt, P., Allard, F., \& Baron, E. 1999, ApJ, 512, 377

Heber, U., Dreizler, S., \& Hagen, H.-J. 1996, A\&A, 311, L17

Hügelmeyer, S. D., Dreizler, S., Werner, K., et al. 2005, A\&A, 442, 309

Krzesiński, J., Nitta, A., Kleinman, S. J., et al. 2004, A\&A, 417, 1093

Leggett, S. K., Hauschildt, P. H., Allard, F., Geballe, T. R., \& Baron, E. 2002, MNRAS, 332, 78

Liebert, J., Wesemael, F., Hansen, C. J., et al. 1986, ApJ, 309, 241

Liebert, J., Bergeron, P., \& Holberg, J. B. 2005, ApJS, 156, 47

McGraw, J. T., Liebert, J., Starrfield, S. G., \& Green, R. 1979, in White

Dwarfs and Variable Degenerate Stars, ed. H. M. Van Horn, \& V. Weidemann

(Rochester: University of Rochester), IAU Colloq., 53, 377

Paczyński, B. 1970, Acta Astron., 20, 47

Quirion, P.-O., Fontaine, G., \& Brassard, P. 2004, ApJ, 610, 436

Schönberner, D. 1983, ApJ, 272, 708

Starrfield, S. 1987, in Second Conference on Faint Blue Stars, ed. A. G. D. Philip,

D. S. Hayes, \& J. Liebert (Schenectady, New York: L. Davis Press), IAU Colloq., 95, 309

Wegner, G. 1979, MNRAS, 187, 17

Werner, K. 1992, Lect. Notes Phys. (Berlin: Springer-Verlag), 401, 273

Werner, K., \& Heber, U. 1991, A\&A, 247, 476

Werner, K., Heber, U., \& Hunger, K. 1991, A\&A, 244, 437

Werner, K., Dreizler, S., Heber, U., et al. 1995, A\&A, 293, L75

Werner, K., Dreizler, S., Heber, U., \& Rauch, T. 1996, in Astrophysics in the

Extreme Ultraviolet, ed. S. Bowyer, \& R. F. Malina (Dordrecht: Kluwer Academic Publ.), IAU Colloq., 152, 229

Werner, K., Deetjen, J. L., Dreizler, S., et al. 2003, in Stellar Atmosphere

Modeling, ed. I. Hubeny, D. Mihalas, \& K. Werner (San Francisco:

Astronomical Society of the Pacific), ASP Conf. Ser., 288, 31

Werner, K., Rauch, T., Napiwotzki, R., et al. 2004, A\&A, 424, 657

Wesemael, F., Green, R. F., \& Liebert, J. 1985, ApJS, 58, 379

Wisotzki, L., Koehler, T., Groote, D., \& Reimers, D. 1996, A\&AS, 115, 227

Wood, M. A. 1995, Lect. Notes Phys. (Berlin: Springer-Verlag), 443, 41

Wood, P. R., \& Faulkner, D. J. 1986, ApJ, 307, 659

York, D. G., Adelman, J., Anderson, J. E., et al. 2000, AJ, 120, 1579

Zhang, E.-H., Robinson, E. L., \& Nather, R. E. 1986, ApJ, 305, 740 